\title{
SULLA RIFRAZIONE DI UNA SUPPOSTA ATMOSFERA LUNARE.
}

NOTA

\section{DEL PRof. F. O. MOSSOTTI.}

Questione. Si domanda se è possibile che un raggio di luce che parte da una stella e traversa ${ }^{\circ}$ l'atmosfera lunare sia rifratta in modo che l'immagine della stella, che esso dipinge, si progelli sopra il disco della luna.

Risposta. Le equazioni del movimento di una molecola Juminosa che penetra un mezzo composto di coppe sferiche concentriche di poter rifrangente variabile, espresso da $k \Delta$, quando si prenda per origine delle coordinate il centro della sfera, sono

$$
\frac{\mathrm{d}^{2} x}{\mathrm{~d} t^{2}}=k \frac{\mathrm{d} \Delta}{\mathrm{d} r} \frac{x}{r} ; \quad \frac{\mathrm{d}^{2} y}{\mathrm{~d} t^{2}}=k \frac{\mathrm{d} \Delta}{\mathrm{d} r} \frac{y}{r} ; \quad \frac{\mathrm{d}^{2} z}{\mathrm{~d} t^{2}}=k \frac{\mathrm{d} \Delta}{\mathrm{d} r} \frac{z}{r}
$$

le quali forniscono direttamente i quattro integrali

$$
\begin{gathered}
x \frac{\mathrm{d} y}{\mathrm{~d} t}-y \frac{\mathrm{d} x}{\mathrm{~d} t}=c \\
x \frac{\mathrm{d} z}{\mathrm{~d} t}-z \frac{\mathrm{d} x}{\mathrm{~d} t}=c^{\prime} \\
y \frac{\mathrm{d} z}{\mathrm{~d} t}-z \frac{\mathrm{d} y}{\mathrm{~d} t}=c^{\prime \prime} \\
\frac{\mathrm{d} x^{2}}{\mathrm{~d} t^{2}}+\frac{\mathrm{d} y^{2}}{\mathrm{~d} t^{2}}+\frac{\mathrm{d} z^{2}}{\mathrm{~d} t^{2}}=k \Delta+v^{2} ;
\end{gathered}
$$

$v$ essendo la velocità della luce nel voto.

Le prime tre moltiplicate rispettivamente per $x,-y$, e $z$ e sommate danno

$$
c x-c^{\prime} y+e^{\prime \prime} z=o
$$

Quest'equazione ci mostra che la trajettoria descritta dal raggio di luce è un piano che passa per l'origine delle coordinale o pel centro della sfera.

Poichè la curva descritta dalla molecola luminosa giace in un piano, prendiamo questo piano per quello delle $x y$; in tal caso $z$ e $\frac{\mathrm{d} z}{\mathrm{~d} t}$ saranno sempre nulle, e si arranno semplicemente le due equazioni

$$
x \frac{\mathrm{d} y}{\mathrm{~d} t}-y \frac{\mathrm{d} x}{\mathrm{~d} t}=c ; \quad \frac{\mathrm{d} x^{2}}{\mathrm{~d} t^{2}}+\frac{\mathrm{d} y^{2}}{\mathrm{~d} t^{2}}=\frac{\mathrm{d} s^{2}}{\mathrm{~d} t^{2}}=v^{2}+k \Delta .
$$


PURA ED APPLICATA.

Permutando le differenziali, così che siano prese relativamente ad $s$ ed eliminando $\frac{\mathrm{d} s}{\mathrm{~d} r}$ fra le due equazioni si avrà

$$
x \frac{\mathrm{d} y}{\mathrm{~d} s}-y \frac{\mathrm{d} x}{\mathrm{~d} s}=\frac{c}{\sqrt{v^{2}+k \Delta}}
$$

Questa equazione è quella che va a fornirci la risposta al quesito.

Chiamiamo $180^{\circ}-\theta$ l'angolo che la tangente della trajettoria fa coll'asse delle $x$, così che sia

$$
\text { (2) } \frac{\mathrm{d} x}{\mathrm{~d} s}=-\cos \theta
$$

(3) $\frac{\mathrm{d} y}{\mathrm{~d} s}=\sin \theta$

e riferiamo la curva alle coordinate polari, ponendo

$$
x=r \cos \varphi \quad y=r \sin \varphi
$$

con queste denominazioni la precedente equazione darà

$$
r \sin (\varphi+\theta)=\frac{c}{\sqrt{y^{2}+\overline{k \Delta}}}
$$

che servirà a determinare la costante arbitraria $c$. In falti è facile di verificare che l'angolo $\varphi+\theta$ è quello che fà la tangente alla trajettoria col raggio $r$; supponiamo che al limite dell'atmosfera dove $k \Delta=0$ ed $r=1+\tau$ sia $\zeta$ il valore di questo angolo, si avrà

$$
(1+\tau) \sin \zeta=\frac{c}{v}, \quad c=v(1+\tau) \sin \zeta
$$

e quindi

$$
r \sin (\varphi+\theta)=\frac{(1+\tau) \sin \zeta}{\sqrt{1+\frac{k}{v^{2}}} \Delta}
$$

Dalle equazioni (2) e (3) si ricava

$$
\begin{gathered}
\frac{\mathrm{d} x}{\mathrm{~d} s}=\left(\cos \varphi-r \sin \varphi \frac{\mathrm{d} \varphi}{\mathrm{d} r}\right) \frac{\mathrm{d} r}{\mathrm{~d} s} ; \quad \frac{\mathrm{d} y}{\mathrm{~d} s}=\left(\sin \varphi+r \cos \varphi \frac{\mathrm{d} \varphi}{\mathrm{d} r}\right) \frac{\mathrm{d} r}{\mathrm{~d} s} \\
x \frac{\mathrm{d} y}{\mathrm{~d} s}-y \frac{\mathrm{d} x}{\mathrm{~d} s}=r^{2} \frac{\mathrm{d} \varphi}{\mathrm{d} r} \frac{\mathrm{d} r}{\mathrm{~d} s} \quad \frac{\mathrm{d} s^{2}}{\mathrm{~d} r^{2}}=1+r^{2} \frac{\mathrm{d} \varphi^{2}}{\mathrm{~d} r^{2}}
\end{gathered}
$$

sostituendo questi valori, e quello di $c$, nell'equazione (1) si avrà finalmente l'equazione della trajettoria del raggio di luce riferito alle coordinate polari, che sarà

$$
\mathrm{d} \varphi=\frac{\mathrm{d} r}{r \sqrt{\frac{\left(1+\frac{k}{v^{2}} \Delta\right) r^{2}}{(1+\tau)^{2} \sin ^{2} \zeta}-1}}
$$


Per semplificare un poco quest'equazione, prendiamo per unità il raggio della sfera solida, o della luna, e supponiamo che $k$ rappresenti il poter rifrangente alla sua superficie cosichè quando $r=1$ si abbi pure $\Delta=1$; considerando $\Delta$ come una funzione di $r$, e posto per brevità, $\frac{k}{v^{2}}=i$, facciamo

$$
\frac{1+i \Delta}{1+i}=\frac{\varphi(r)}{r^{2}}
$$

Con queste posizioni l'equazione precedente prenderà la forma

$$
\mathrm{d} \varphi=\frac{\mathrm{d} \log r}{\sqrt{\varphi(r)-\frac{(1+\tau)^{2}}{1+i} \sin ^{2} \zeta}}
$$

Consideriamo un raggio di luce che parte da un punto della superficie lunare, e sorte dall'atmosfera. Sia $M$ il minimo valore che può avere la funzione $\varphi(r)$ in tulta l'estensione dell'atmosfera; affinchè il radicale della precedente equazione si mantenga sempre reale, ed il raggio di luce possa di fatto uscire, dovrà essere

$$
\frac{(1+\tau)^{2}}{1+i} \sin ^{2} \zeta<M
$$

Dunque il più gran valore di $\sin ^{2} \zeta_{0}$, solto il quale un raggio di luce, che parte dalla superficie della luna, puo sortire dall'atmosfera, sarà dato dall'equazione

$$
\sin ^{2} \zeta_{0}=\frac{i+\tau}{(1+\tau)^{2}} \mathbf{M} \text {. }
$$

Suppongasi ora che un raggio di luce entri nell' atmosfera lunare per atraversarla e sortire dalla parte opposta: Questo raggio nel suo corso si avvicinerà al centro della luna sino ad un certo punto per poi allontanarsi, di modo che $r$ avrà un minimo, e sarà per questo punto $\frac{\mathrm{d} r}{\mathrm{~d} \varphi}=0$, dunque per questo valore l'equazione (3) darà

$$
\varphi(r)=\frac{(1+\tau)^{2}}{1+i} \sin ^{2} \zeta
$$

a) sia

$$
\sin ^{2} \zeta=\frac{1+i}{(1+\tau)^{2}} \varphi(r)
$$

Ma il secondo membro ha per valore minimo $\sin ^{2} \zeta_{0}$ dunque dovrà sempre essere

$$
\sin ^{2} \zeta>\sin ^{2} \zeta_{0}
$$

e come i due raggi di luce non possono uscire dall'almosfera della stessa parte delle 
ascisse positive se $\sin \zeta$ e $\sin \zeta_{0}$ non hanno lo stesso segno, perciò la precedente equazione darà anche

$$
\dot{\xi}>\zeta_{\mathrm{o}}
$$

Dunque un raggio che traversa l'atmosfera non può mai al sortire fare col raggio vetlore un angolo più piccolo del più grand' angolo che un raggio che parte dalla superficie lunare farà col rispetto raggio vettore nel punto d'emergenza.

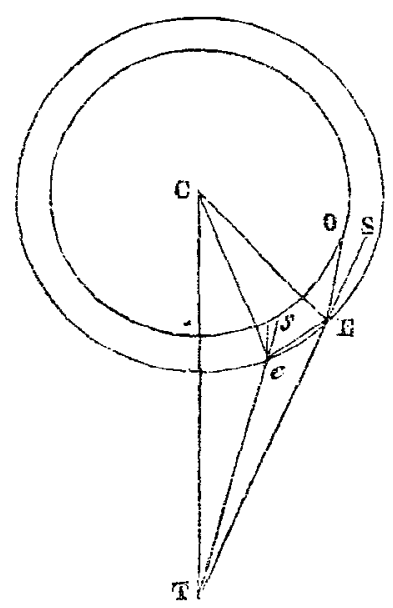

Ciò posto sia $\mathrm{T}$ il luogo dell'osservatore sulla terra, $\mathrm{C}$ il centro della luna, TS il raggio che parte dal punto $O$ e costituisce l'immagine del punto più esteriore del lembo della luna e sorte sotto il più grand'angolo CES il cui seno ha per espressione. $\frac{\sqrt{1+i}}{1+\tau} \sqrt{\mathrm{M}}$. Se un altro raggio di luce $\mathrm{T} s$ avesse potuto traversare l'atmosfera e dipingere l'immagine della stella sul disco della luna, dovrebbe essere l'angolo CTs < CTS, o sia il punto $e$ dovrebbe cadere interiormente nel triangolo CTE. Ora nel triangolo CET si ha l'angolo esterno CES = ECT + CTE e nel triangolo $\mathrm{C} e \mathrm{~T}$ si ha $\mathrm{C} e s=e \mathrm{CT}+\mathrm{CT} e$, ma ̀̀ $\mathrm{ECT}>e \mathrm{CT}$, e $\mathrm{CTE}>\mathrm{CT} e$, dunque dovrebbe essere $\mathrm{CES}>\mathrm{Ces}$, cioè l'angolo che il raggio di luce $\mathrm{OET}$, che parte da un punto della superficie della luna, fa col raggio $\mathrm{CE}$ al punto d'emergenza, dovrebbe essere maggiore dell'angolo che il raggio di luce se $\mathbf{T}$ che viene dalla stella ed ha traversato l'atmosfera fa col rispettivo raggio $\mathrm{C} e$, la qual cosa, come abbiamo veduto sopra non è possibile.

$N$. Questa breve Nota fù scritta dal Prof. Mossotti in lioma nel 1836, e la lasciò in mie mani. Ho creduto ora di pubblicarla, pensando di far cosa grata ai lettori, quando ad essi si rammenti l'insigne Scienziato recentemente perduto.

B. 'T.

Tom. V. N. 2. 